Britta König*, Nika Guberina, Hilmar Kühl, and Waldemar Zylka

\title{
Design and first results of a phantom study on the suitability of iterative reconstruction for lung-cancer screening with low-dose computer tomography
}

https://doi.org/10.1515/cdbme-2019-0149

\begin{abstract}
In this research computer tomography (CT) iterative reconstruction (IR) algorithms are investigated, specifically the impact of their statistical and model-based strength on image quality in low-dose lung screening CT protocols in comparison to filtered back projection (FBP). It has been probed whether statistical, model-based IR in conjunction with low-dose, and ultra-low-dose protocols are suitable for lungcancer screening. To this end, artificial lung nodules shaped as spheres and spicules made from material with calibrated Hounsfield units (HU) were attached on marked positions in the lung structure of an anthropomorphic phantom. Nodule positions were selected by distinguished radiologists. The phantom with nodules was scanned on a CT Scanner using standard high contrast (SHC), low-dose (LD) and ultra low-dose (ULD) protocol. For reconstruction FBP and the IR algorithm ADMIRE at three different strength levels were used. Volume CT dose index (CTDIvol) and dose-length product were recorded. Radiologists assessed subjective image quality using a six-point Likert scale by reading all image series in terms detectability of lung nodules. As a measurable objective image quality parameter signal-to-noise ratios (SNR) were investigated. The CTDIvol decreases by more than $70 \%$ for all protocols and nodules compared to diagnostic reference value for chest $\mathrm{CT}(\mathrm{p}<0.00001)$. The evaluation of image quality parameters, i.e. SNR, indicates that LD and ULD protocols in conjunction with IR assert high quality lung-nodule detection. The results reveal that IR algorithm with moderate to high strength is an indispensable alternative to FBP in low-dose scanning, thus, potentially suitable for lung-tumour screening.
\end{abstract}

\footnotetext{
*Corresponding author: Britta König, Westphalian University, Campus Gelsenkirchen, Germany, and University of Duisburg-Essen, britta.koenig@stud.uni-due.de Nika Guberina, University of Duisburg-Essen, University Hospital Essen, Germany, nika.guberina@uk-essen.de Hilmar Kühl, St. Bernhard-Hospital Kamp-Lintfort GmbH, University of Duisburg-Essen, Germany, hilmar.kuehl@uni-due.de Waldemar Zylka, Westphalian University, Campus Gelsenkirchen, Germany, waldemar.zylka@w-hs.de
}

Keywords: CT, iterative reconstruction, FBP, low-dose, image quality, phantom study, lung nodule detection

\section{Introduction and Background}

The Center for Cancer Registry Data predicted 33700 new cases of lung cancer among men and 22000 among women in Germany in 2018. According to the US Lung Cancer Screening Trial (NLST) in 2011, the low-dose computer tomography screening procedure could reduce the relative risk of dying of lung cancer in the risk group by $20 \%$, which corresponds to an absolute risk reduction of $0.3 \%$ [1,2]. To detect lung nodes, high contrast CT scanning could be used in conjunction with low dose. Manufacturers offer several approaches to keep the delivered dose as low as possible: Reduction of tube voltage, automatic tube current modulation and iterative reconstruction are only the most recent techniques [3]. However, when using the filtered back projection (FBP) for image reconstruction, the low-dose CT displays a poor image quality and thus may negatively influence the diagnostic performance of readers.

One of the main advantages of IR reconstruction algorithms is their inherent discrete mathematical formulation. This allows to model the complete CT acquisition process, e.g. including detector geometry and sensitivity, beam shape, physics of the $\mathrm{x}$-ray quanta as well as the incorporation of prior knowledge like noise characteristics and electronic noise. The new generation of IR reconstruction approaches uses statistical and model-based methods. Statistical methods incorporate e.g. counting statistics of detected photons and can be used in the raw and in the image data domain or during the reconstruction process. Model-based methods go even beyond the statistical modeling by embedding geometric models of the components of the CT scanner.

In this investigation Advanced Modeled Iterative Reconstruction (ADMIRE), the currently most recent iterative reconstruction approach of Siemens, is used. Compared to FBP, the perception of ADMIRE reconstructed low-dose CT images is different and increases with increasing strength of the algorithm. The reservations of radiologists about the changed image impression and its possible influence on diagnostic perfor- 
mance with regard to the detection of lung nodes in low-dose CT are an incentive for a comparative investigation of objective and subjective image quality factors and diagnostic performance. Thus, this paper reports on a phantom study which probed whether FBP and the statistical model-based iterative reconstruction of the ADMIRE approach in conjunction with high-contrast, low-dose, and ultra-low-dose protocols might be suitable for lung-cancer screening.

\section{Materials and methods}

Antrophomorphic Phantom. The anthropomorphic multipurpose N1 LUNGMAN phantom PH-1 R16511 (Kyoto Kagaku Co.,Ltd., Kyoto, Japan) was used to replicate an adult human chest. The life-size anatomical model consits of components with calibrated Hounsfield values (HU) of human tissue. In particular, chest wall with synthetic bones, pulmonary vasculature with mediastinum (trachea and heart) and diaphragm abdominal block. To simulate pulmonary leasions we used artifical lung nodules (QRM GmbH, Moehrendorf, Germany) shaped as spheres with diameters $3 / 5 / 8 / 10 / 12 \mathrm{~mm}$ and spicules with diameters $16 / 20 / 24 \mathrm{~mm}$, both made from material with calibrated (at $120 \mathrm{kV}$ ) HU values equal to $-690 /-50 /+100$, see Fig. 1(b). Tissue equivalent materials for the human habitus, inner components and nodes are made from polyurethane, urethane foam, epoxy resin and calcium carbonate. Nodules, i.e spheres and (in a second setup) spicules, were glued with gel on marked positions displayed Fig. 1(c)-(d). These positions were selected by distinguished radiologists. We placed the phantom surpine and head first, as shown in Fig. 1(a).

CT protocol and reconstruction. All scans were acquired on a Somatom Force CT (Siemens Healthineers GmbH, Erlangen, Germany) at the University Hospital Essen (Essen, Germany). It is equipped with a Vectron ${ }^{\mathrm{TM}} \mathrm{X}$-ray tube and a Stellar ${ }^{\text {Infinity }}$ detector with anti-scatter 3D collimator grid. The detector acquisition configuration was $192 \times 0,6 \mathrm{~mm}$. The use of z-flying focal spot technique enable double sampling along the $\mathrm{z}$-axis. Three dose protocols were selected: (i) standard high contrast (SHC; $120 \mathrm{kV} /$ dose modulation), (ii) low-dose (LD; 120kV/40mAs) and (iii) ultra-low-dose (ULD; $120 \mathrm{kV} / 20 \mathrm{mAs}$ ). Raw data were acquired using FOV 300mm, rotation time $0.5 \mathrm{~s}$, delay $2 \mathrm{~s}$, beam collimation $192 \times 0.6 \mathrm{~mm}$, deactivated CAREDose4D, slice thickness $1 \mathrm{~mm}$, increment $1 \mathrm{~mm}$. Preparations scans were performed using Waterphantom_comp_MxT Modeltype 10742380 (Siemens Healthineers $\mathrm{GmbH}$, Erlangen, Germany). Images were reconstructed as $1 \mathrm{~mm}$ axial slices with FBP and ADMIRE, strength S3, to ensure the scanner calibration and to verify the stability of reconstruction process.

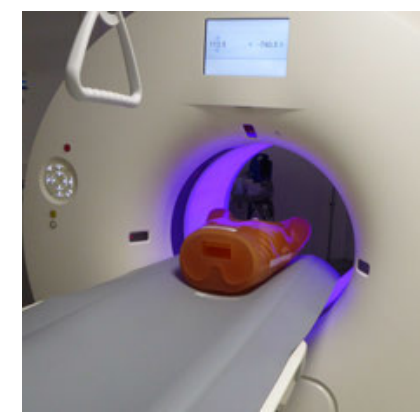

(a) LUNGMAN phantom inside CT

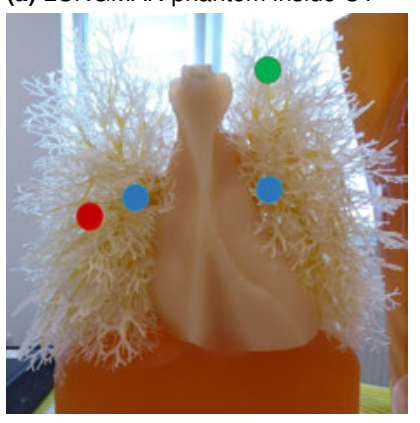

(c) Ventral view with marked positions

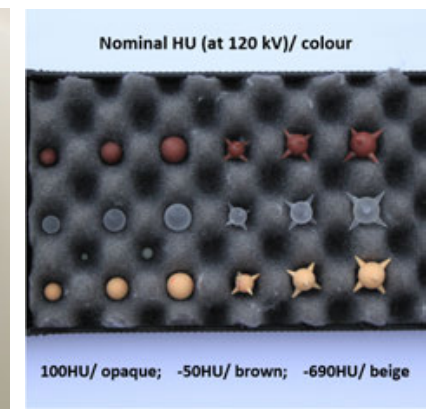

(b) Artifical lung nodules

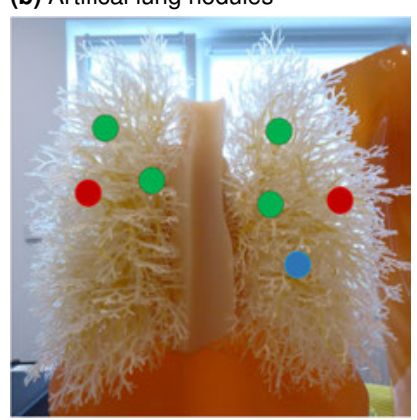

(d) Dorsal view with marked positions

Fig. 1: Phantom within the CT-Gantry (a), artificial lung nodules (b), and their positions in ventral (c) and dorsal (d) view of the pulmonary vasculature. Color assignment: green $100 \mathrm{HU}$; blue $-50 \mathrm{HU}$; red $-690 \mathrm{HU}$.

The acquired raw data of each protocol were reconstructed by utilizing three kernels: BL57 is a standard for lung node detection, BR32 is a soft, and BR69 is hard kernel. The images were reconstructed using FBP and the iterative algorithm ADMIRE with strength S1, S3, and S5 as axial slices of $5 \mathrm{~mm}$ thickness, $5 \mathrm{~mm}$ reconstruction increment with parenchymal lung window (-600/1200HU). In total seventy-two CT image series each consisting of sixty-two images were acquired. For the assessment of the signal-to-noise ratio (SNR) slice twenty-nine of each CT series is selected and subsequently these collection of slices were anonymized and randomized.

Evaluation methods. The assessment of the dose is based on the applied CT dose protocol. Volume CT dose index, CTDIvol, and dose-length product DLP were recorded for each acquisition and the effective dose was estimated by multiplying the DLP by a chest-specific conversion coefficient $0,014 \mathrm{mSv} \mathrm{mGy}^{-1} \mathrm{~cm}^{-1}$. For every CT acquisition the CTDIvol results were compared to the diagnostic reference value for chest CT (10mGy) issued by the Federal Office for Radiation Protection, Germany, [5]. Student's t-Test (paired, one sided) was invoked to calculate statistically significant differences (p-values). Signal-to-noise ration (SNR) was investigated as measurable objective image quality parameter. Four 
specific landmarks were defined: in the periphery above the sternum into air (LP), in the right lung vasculature (LR), in the left lung vasculature (LL), and in tissue placed right paravertrebral (LT). In the previously defined collection of images a region of interest (ROI) was drawn on every landmark with the area of $(1.00 \pm 0.05) \mathrm{cm}^{2}$ was selected and the mean CT value, standard deviation and pixel value were calculated. This investigation was carried out independently by four medical engineers who used RadiAnt Dicom Viewer (Medixant, Poznan, Poland). Four radiologists with different professional experience in thoracic diagnosis reviewed all CT series extensively in terms of lung nodule detection. They use an approved clinical diagnostic monitor and Software. The readers assessed subjective perceptibility of the lung nodes in percent measured on six-point Likert scale.

\section{Results}

The CT dose protocols for SHC, LD and ULD scans with spheres and spicules, as well as the estimated effective dose are listed in Tab. 1. Compared to the diagnostic reference value for chest CT, the CTDIvol drop for spherical targets is $-70.1 \%$ for $\mathrm{SHC},-73.5 \%$ for $\mathrm{LD}$, and $-86.9 \%$ for ULD. Considering spicules, the CTDIvol decreases by $-69.9 \%$ for SHC, $-73.5 \%$ for $\mathrm{LD}$, and $-89.9 \%$ for ULD ( $\mathrm{p}<0.00001)$.

Tab. 1: Dose delivered by standard high contrast (SHC), low-dose (LD), and ultra-low-dose (ULD) scans for spheres and spicules

\begin{tabular}{rrrr}
\hline $\begin{array}{r}\text { scan } \\
\text { protocol }\end{array}$ & $\begin{array}{r}\text { CTDlvol } \\
\text { [mGy] }\end{array}$ & $\begin{array}{r}\text { DLP } \\
\text { [mGycm] }\end{array}$ & $\begin{array}{r}\text { Effective Dose } \\
\text { [mSv] }\end{array}$ \\
\hline SHC sphere & 2,99 & 106,30 & 1,49 \\
LD sphere & 2,65 & 94,00 & 1,32 \\
ULD sphere & 1,31 & 46,50 & 0,65 \\
\hline SHC spicules & 3,01 & 106,90 & 1,50 \\
LD spilcules & 2,65 & 94,00 & 1,32 \\
ULD spicules & 1,31 & 46,50 & 0,65 \\
\hline
\end{tabular}

For each protocol, the ROI measurement in the left lung generated the ROIs mean $\mathrm{HU}$ value $\mu_{i}$, standard deviation $\sigma i$ and the number of pixels, in each of seventy-two images. The $\mathrm{SNR}_{i}$ of one ROI was calculated according to Eq.(1). The $\mathrm{SNR}_{i}$ values with the same index $i$ but from the different test protocols are presented in Figure 2 for spheres and spicules.

$$
\mathrm{SNR}_{i}=\left|\frac{\mu_{i}}{\sigma i}\right| \text {. }
$$

The SNR for the standard BL57 kernel ranges between 3.390 for spheres and 7.102 for spicules, ULD and S5. The

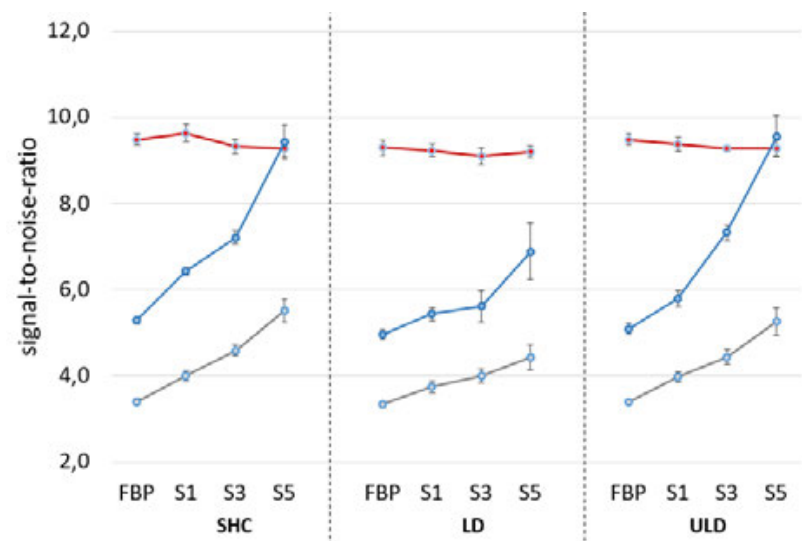

(a) Spheres

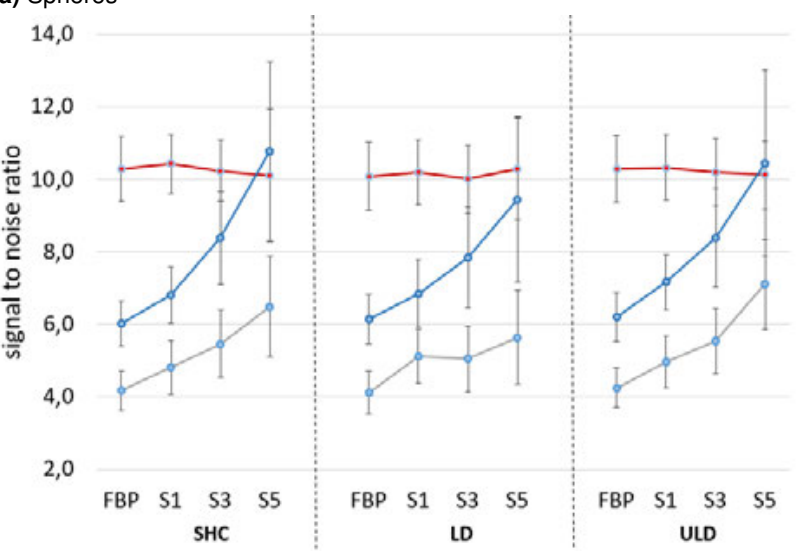

(b) Spicules

Fig. 2: Averaged SNR in the left lung landmark for spheres (a) and spicules (b) for various kernels: soft BR32 (red), standard BL57 (gray), hard BR69 (blue). The error bars indicate the standard deviation of the mean.

SNR interval of hard BR69 over all protocols is between 4.963 (LD, spheres, FBP) and 10.763 (SHC, spicules, S5). Both kernels show higher SNR with increasing algorithm strength. SNR is lowest for reconstruction with FBP. The BR32 kernel shows higher SNR values than other kernels except S5 in SHC and ULD for spheres and spicules. SNR maximum for the soft BR32 kernel is 10.425 for spicules, SHC and S1 while the lower SNR limit is 9.101 for spheres with S3. The soft BR32 kernel has the smallest SNR interval. The highest SNR single value of 10.763 is observed for BR69 kernel whe imaging spicules in the SHC protocol an iteration strength S5. The SNR values and the standard deviation of the mean of spicules are higher compared to the spheres.

Results of the Likert scale assessment for the spherical nodules are presented in Fig. 3. The radiologist rated the perceptibility for the standard kernel BL57 with "good/moderate" and with "moderate/sufficient" for the soft kernel BR32 . FBP was graded with the highest occurring perceptibility for each 

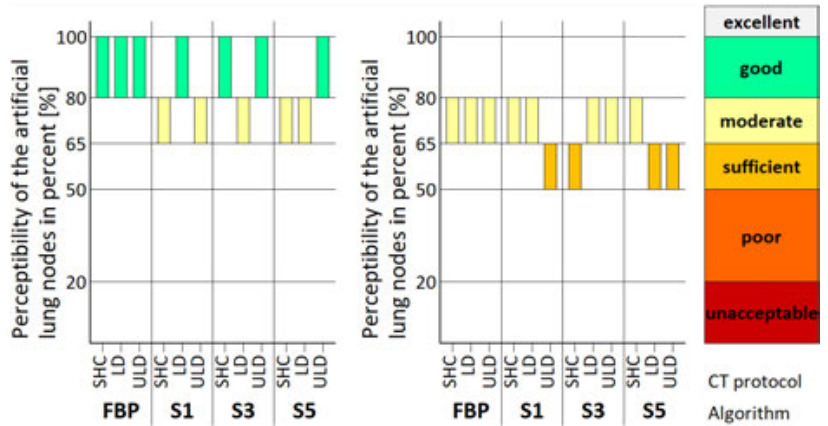

Fig. 3: Perceptibility of artificial lung nodes in percent using the six-point Likert scale. The results for spheres are displayed for the reconstruction kernels BL57 (left) and BR32 (right) using FBP and IR algorithms and dose protocols SHC, LD and ULD.

kernel. The grade "sufficient" occurred for the kernel B32 with IR strength S1-S5 at least once and most often in conjunction with ULD. Notably, overall radiologist's perception prefer the standard B57 kernel. The ratings "excellent" and "unacceptable" were not assigned in any case.

\section{Discussion and Conclusion}

This study suggest that low-dose and ultra-low-dose protocols for lung node detection in connection with model-based iterative reconstruction with moderate to high strengths could be an essential alternative to FBP. For the standard kernel BL57 subjective image quality ranged from "good" to "moderate" in all images and displays only a slight loss of quality compared to the consistently "good" FBP assessment.

In [6] various IR algorithms in conjunction to low-contrast detectability in abdominal computed tomography are reported and it was concluded that switching form FBP to IR offers limited possibilities to achieve significant dose reductions while maintaining a constant objective low-contrast detectability. We suspect the limits does not apply for high-contrast lung node detection. A detailed comparison of Likert scale results of a radiologist with six years diagnostic experience to those of a reader with three years of professional practice indicate that radiologists with many years of professional practice may be more influenced and used to read FBP reconstructed images. In order to be able to provide more accurate information regarding the subjective image quality and diagnostic performance, the evaluation of further radiologists and statistics are necessary.

The benefit of increasing SNR in low-dose and ultra-lowdose protocols with the kernel BL57 used in clinical environment and increasing IR strength was expected reasoned by op- eration mode of model-based IR. It can be assumed that the soft kernel BR32 is less susceptible to influence as it is designed to operate at lower frequencies. In order to make statements about the SNR influence of individual kernels in LD and ULD protocols, especially in case of BR32, further measurement protocols will be evaluated and additional statistical analysis is in progress. SNR differences between spheres and spicules could be substantiated by offsets caused by slightly different placement of spheres and spicules.

Our results for iterative reconstruction in conjunction with artificial nodules placed in phantoms compare to [3]. Concerning dose reduction and subjective image quality, our preliminary results reveal that IR algorithm with moderate to high strength is advisable for lung-cancer screening.

\section{Author Statement}

Research funding: The author state no funding involved. Conflict of interest: Authors state no conflict of interest.

\section{References}

[1] Center for Statistical Sciences, Brown University,Providence, United States of America. The National Lung Screening Trial: Overview and Study Design. Radiology 2011;258:243-253.

[2] Robert Koch Institute. The Center for Cancer Registry Data (DE). Lunge-C33-34 Krebs in Deutschland https://www.krebsdaten.de/Krebs/DE/Content/Publikationen /Krebs_in_Deutschland/kid_2017/kid_2017_c33_c34_lunge.pdf? blob=publicationFile. Last accessed 2017-12-06.

[3] Macri F., Greffier J., Pereira F.R., Mandoul C., Khasanova E., Gualdi G., et al. Ultra-low-dose chest CT with iterative reconstruction does not alter anatomical image quality. Diagnostic and Interventional Imaging 2016;97:1131-1140.

[4] Beister M., Kolditz D., Kalender W.A.. Iterative reconstruction methods in X-ray CT. Physica Medica 2012;28:94-108.

[5] Federal Office for Radiation Protection (DE). Bekanntmachung der aktualisierten diagnostischen Referenzwerte. http://www.bfs.de/DE/themen/ion/anwendungmedizin/diagnostik/referenzwerte/referenzwerte_node.html Last accessed 2018-08-21.

[6] Viry A, Aberle C., Racine D., Knebel J-F., Schindera S.T., Schmidt $S$, et al..Effects of various generations of iterative CT reconstruction algorithms on low-contrast detectability as a function of the effective abdominal diameter: A quantitative task-based phantom study. Physica Medica 2018;48:111-118. 